Proceedings of the XXIII Conference on Applied Crystallography, Krynica Zdrój, Poland, September 20-24, 2015

\title{
Phononic and Phasonic Debye-Waller Factors for 1D Quasicrystals
}

\author{
J. Wolny*, I. Buganski And R. Strzalka \\ AGH University of Sciences and Technology, Faculty of Physics and Applied Computer Science, \\ al. A. Mickiewicza 30, 30-059 Krakow, Poland
}

\begin{abstract}
The main purpose of crystallography is to solve and refine crystal structures based on measured diffraction data. One of important corrections crucial in the refinement process is the Debye-Waller factor correction for phonons in physical, and phasons in perpendicular space. In our paper we show the limitations of the standard approaches to the Debye-Waller correction in case of quasicrystals and propose new approach based on the statistical method. For the model 1D quasicrystal we show that in case of phonons there is no significant objection against classical (exponential) Debye-Waller factor, however using different forms can slightly improve the results of a refinement. In case of phasons the classical formula gives no rise to the efficiency of the refinement and completely new approach is required. We propose a redefinition of the Debye-Waller factor in terms of the statistical approach and show its effectiveness.
\end{abstract}

DOI: 10.12693/APhysPolA.130.836

PACS/topics: 61.44.Br, 61.05.cc, 61.50.Ah

\section{Introduction}

The Debye-Waller correction in general compensates for the perturbations of the atomic positions from their ideal values during the refinement process. Thermal vibrations of atoms known as static or "frozen" phonons is the most significant phenomenon to be considered. It gives rise to a reduction of the peaks height in the diffraction pattern. This effect is more clearly seen for peaks with large scattering vector. The most common form of the compensating factor is given by a Gaussian approximation and called Debye-Waller factor $(\mathrm{D}-\mathrm{W})$. It is expressed by exponential function of mean-square displacement of atoms $\left\langle x^{2}\right\rangle$ multiplied by the squared scattering vector $\boldsymbol{k}$. If the displacement of non-equivalent atoms does not depend on $\boldsymbol{k}$, we define an isotropic D-W factor, which introduces a single parameter for a whole crystal during the refinement. The formula for isotropic $\mathrm{D}-\mathrm{W}$ factor is the following [1]:

$$
\begin{gathered}
D_{\text {phon }}(\boldsymbol{k})=\exp \left(-\frac{1}{2}\left\langle x^{2}\right\rangle \boldsymbol{k}^{2}\right)= \\
\exp \left(-\frac{1}{16 \pi^{2}} \boldsymbol{k}^{2} b_{\text {phon }}\right),
\end{gathered}
$$

where $b_{\text {phon }}=8 \pi^{2}\left\langle x^{2}\right\rangle$ is the isotropic atomic displacement parameter (ADP). For model 1D case the ADP can only be isotropic. In further parts of the paper by classical D-W factor for phonons we mean the formula (1).

More realistic case in real structure refinement is the dependence of $\left\langle x^{2}\right\rangle$ on the direction of $\boldsymbol{k}$. Parameters $b_{\text {phon }}$ become anisotropic ADPs. The matrix representing anisotropic ADPs is symmetric with only six independent parameters. Beside the Gaussian approximation also anharmonic terms to the exponent can sometimes be considered. Most widely used is the cumulant or quasi-moment (Gram-Charlier) expansion recommended by the IUCr [1].

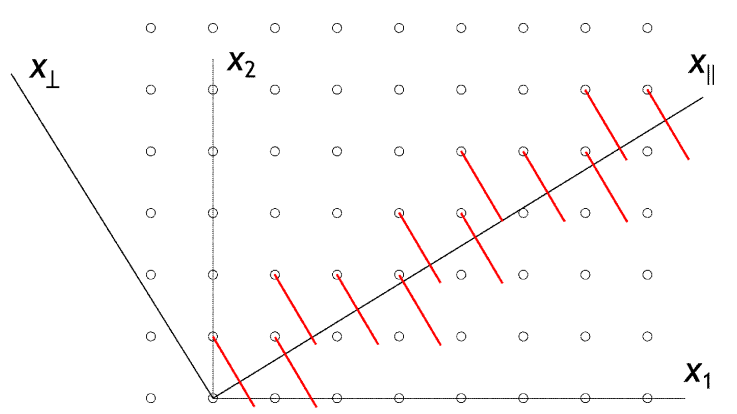

Fig. 1. Higher-dimensional representation of the Fibonacci chain. Atomic surfaces (red segments) spanned along perpendicular-space direction $\left(x_{\perp}\right)$ represent atoms in embedded 2D space. The atomic structure in physical space can be obtained by a cut along $x_{\|}$.

Another class of imperfections of real crystals caused by atomic displacements are phasons (or phason modes). Their special role in quasicrystals is being discussed since the early stage of research on this type of materials. For quasiperiodic systems the configurational disorder is observed even at zero-temperature, where the atoms are ordered and no thermal vibrations occur. In such case the atomic flips from one position to another are observed independently of phonons. It is highly expected that phasons as entropic phenomenon are crucial in terms of stabilization process in quasicrystals. This long-term investigation is widely discussed in the literature $[2,3]$. Whereas the physics of phonons is clear, the nature of phasons cannot be simply understood by classical consideration. The most successful description of phasons is derived by higher-dimensional analysis and, more precisely, by considering the perpendicular-space component of the hyperspace crystal. Quasiperiodic structure can be understood as periodic in high dimensions and it is modelled in perpendicular space, where atoms are replaced by multi- 
dimensional objects called atomic surfaces (or occupation domains, Fig. 1). Atomic structure is obtained by a projection of atomic surfaces from high dimensions. Phasons are introduced by shift of the projection strip or, equivalently, tilt of the cutting plane [4]. Such a distortion in perpendicular space results in flips of atoms in physical space, which is called phason flips. Phasons were also discussed within a hydrodynamic theory $[5,6]$. Despite the different physical background, the correcting factor for phasons was, however, simply transferred from theory of phonons in crystals. It is also considered as exponential term with perpendicular-space scattering vector $\boldsymbol{k}^{\perp}$ instead of physical-space one $\boldsymbol{k}$. Most frequently the isotropic factor is used introducing a single parameter in the refinement. By analogy to phonons, the compensation for phason flips is obtained by the perpendicularspace (or phasonic) Debye-Waller factor which reads

$$
D_{\text {phas }}\left(\boldsymbol{k}^{\perp}\right)=\exp \left(-\frac{1}{16 \pi^{2}}\left(\boldsymbol{k}^{\perp}\right)^{2} b_{\text {phas }}\right),
$$

where $b_{\text {phas }}$ denotes a parameter to be refined.

We prove that the classical phasonic D-W factor (exponential term) works correctly only for small values of the exponent $\left(\boldsymbol{k}^{\perp}\right)^{2}$ in formula (2) which essentially means the strong diffraction peaks. Including weak reflections in a refinement procedure frequently makes the refinement results worse which can be seen in many refinements (see e.g. $[7-9]$ ). We show how to improve the use of $\mathrm{D}-\mathrm{W}$ factor for phasons and phonons in quasicrystals. Our calculations are performed for a simple 1D model quasicrystal - the Fibonacci chain. The Fibonacci chain can be constructed with two segments $L$ and $S$ of lengths ratio $L / S=\tau(\tau=1.618 \ldots$ is a golden mean) decorated with atoms in the nodes. The sequence of $\{\mathrm{L}, \mathrm{S}\}$ segments follows the Fibonacci recurrence rule, which makes it quasiperiodic but perfectly ordered. The diffraction pattern of Fibonacci chain, although aperiodic, consists of sharp Bragg peaks grouped in periodic series [10].

\section{Method}

The method we use in structure modeling of quasicrystals is based on the statistical approach, where instead of the atomic surfaces in perpendicular space the statistical distributions of projections of atoms onto reference lattice is introduced $[11,12]$. Because of aperiodicity of quasicrystals two reference lattices are needed and two parameters of the distribution are introduced $(u, v)$. Parameters $(u, v)$ define a position of projection of atoms onto the reference lattices. Total statistical distribution $P(u, v)$ of positions $(u, v)$ is non-zero only along a segment line $v=-\tau^{2} u$, which is known as TAU2scaling [13]. The marginal distribution $P(u)$ (called an average unit cell) is dense and uniform and it exists in physical space. A structure factor formula can be calculated as a Fourier transform of the average unit cell as follows $[11,14]$ :

$$
F(k)=\int_{\mathrm{AUC}} P(u) \exp \left(\mathrm{i} k_{0}(n-m \tau)\right) \mathrm{d} u,
$$

where $k=k_{0}(n-m \tau), k_{0}$ is main scattering vector and $n, m$ are integers.

Statistical method is an alternative to higher dimensional analysis but it is more general (see scheme in Fig. 2). It successfully describes not only periodic crystals or quasicrystals $[15,16]$, but also modulated and composite structures as well as aperiodic structures with singular continuous components in the diffraction pattern [17]. In general it can be applied to arbitrary structures [14, 18].

\section{Higher-dimensional analysis}

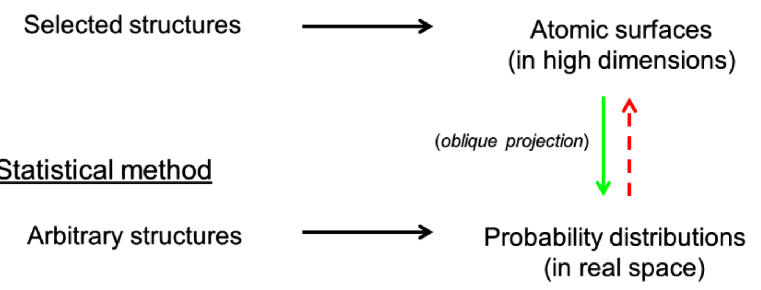

Fig. 2. Scheme of similarities and differences between higher-dimensional (commonly used) and statistical methods. The latter can be applied to arbitrary structures. Probability distributions of atomic positions in the reference lattice frame (in physical space) can be easily obtained by an oblique projection of the atomic surfaces from high dimensions, whereas the physicalspace description cannot be straightforwardly lifted to higher-dimensional one in the most general case.

\section{Phonons}

Phonons straightforwardly appear in the scaling relation (Figure 3) as a characteristic smearing of a segment line. We have investigated one type of phonons described by a Gaussian function generating the displacement of atoms from ideal positions. The amplitude of a displacement is small and it is $\approx 2 \%$ of the average distance $\lambda_{k}$ between atoms (for the Fibonacci chain it is $\lambda_{k}=1+\frac{1}{\tau^{2}} \approx 1.38$ ). Atomic displacements contribute to the shape of the distribution $P(u)$, which clearly deviates from its ideal shape - the uniform distribution for quasicrystals (see Fig. 4a). The function discussed in direct space has its counterpart in Fourier space which is interpreted as a correction function for phonons (D$\mathrm{W}$ factor in the formula for structure factor in reciprocal space). For Gaussian function in direct space we obtain obviously an exponential $\left(\approx \exp \left(-k^{2} \sigma^{2}\right)\right.$, Fig. $\left.4 \mathrm{~b}\right)$. The phononic D-W factor compensates for atomic displacements which manifests in change of calculated (refined) diffraction intensities. We investigated the influence on phonons also by different generating functions (harmonic or flat) and by this different correction functions to the diffraction data (Bessel and cardinal sine functions) were developed. Also, the comparison with periodic crystals was discussed. For details see [19]. Here we focus on the 
Fibonacci chain and standard exponential D-W factor. From Fig. 4b we see that data points (diffraction peaks for a structure with phonons) arrange perfectly on the shape of Gaussian function assumed before. The conclusion is the following: Gaussian D-W correction satisfactorily well compensates for phonons in quasicrystals. It can be successfully used as a standard correction factor in the refinement process. Only at the very last step of the refinement other corrections (e.g. by the Bessel or cardinal sine functions) can be checked for better results.
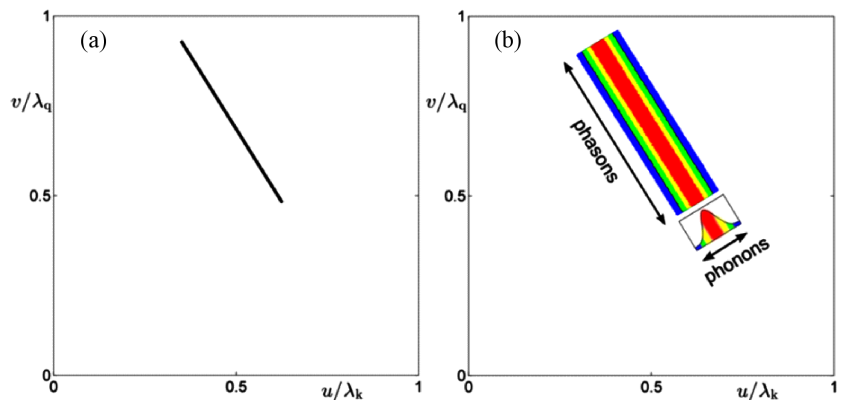

Fig. 3. (a) The TAU2-scaling relation for Fibonacci chain is given by a segment line $v=-\tau^{2} u$. (b) Phonons introduce a spread of a line (if exponential approximation is used, the spread is given by a Gaussian). Phasons introduce change of a distribution along a scaling line (not indicated in coloring, compare with Fig. 5b).
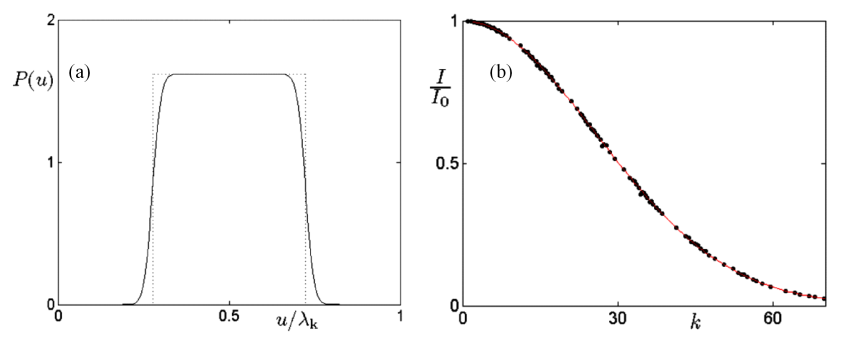

Fig. 4. (a) The influence of a Gaussian function generating phonons on the shape of the average unit cell for the Fibonacci chain - ideal shape (dotted line) and modified shape (solid line) $\left(\lambda_{k} \approx 1.38 \AA\right.$ ). (b) Ratio of intensities with phonons $(I)$ and without phonons $\left(I_{0}\right)$ as a function of the scattering vector for the Fibonacci chain.

\section{Phasons}

In our model calculations we introduced phasons as flips of segments $L S \rightarrow S L$ in the Fibonacci chain with a given probability $\alpha$ (called a flip ratio). Flip ratio defines a percentage of flipped $L S$ sequences with respect to all $L S$ sequences appearing in original chain. Phason flips modify substantially a distribution $P(u)$ (see Fig. 5b). We are able to define strictly which part of the average unit cell $P(u)$ is changed under phasons and what is the change. This gives a full control in real space over the perturbation of atomic positions in the case of phasons. The diffraction pattern can be calculated using structure factor defined in formula (3) with a deformed
$P(u)$. In Fig. 5a and $\mathrm{c}$ we show $\log -\log$ plots of calculated vs. observed intensities for $7 \times 10^{5}$ atoms in the Fibonacci chain (calculated numerically) and flip ratio of $5 \%$ ( $\sim 6500$ diffraction peaks in total). Intensities are scaled to the strongest peak in the data. If no correction for phasons is done (Fig. 5a), clear deviation from a straight line is observed for whole range of intensities. The correction given by the classical D-W formula (2) gives no essential improvement to a refinement result [20]. On the contrary, it introduces a constant deviation from a straight line and leads to the underestimation of calculated intensities (characteristic tail in the plot). This is a known peculiarity of modern refinement results for real quasicrystals. Classical D-W factor is not enough to properly solve a problem of phasons. Much more successful results can be obtained by using different from exponential corrections. As an example we propose the D-W factor defined as a sum of cardinal sine functions:

$$
D_{\text {phas }}(k)=\sum_{i=1,3} A_{i} \operatorname{sinc}\left(\left(k-m k_{1}\right) d_{i}\right)
$$

with parameters $A_{i}$ chosen appropriately $\left(A_{i}\right.$ are complex in general). Parameters $d_{i}$ define widths of three regions of the distribution $P(u)$ (see Fig. 5b). Additional parameters are directly related to flip ratio, hence they have clear physical interpretation. The ratio of flips is proportional to area of fragments (of widths $d_{i}$ and heigths $A_{i}$ ) relocated with respect to ideal distribution $P(u)$. Parameters $d_{i}$ are directly related to the type of flip $(L S \rightarrow S L$ or inversely) and are constant for different flip ratios. Therefore, the flip ratio can be straightforwardly obtained from parameters $A_{i}$, at given type of flips. The result of a refinement with phason correction given by formula (4) is shown in Fig. 5c. No deviation from a straight line is observed and the fit is nearly perfect, being affected only by numerical errors. All refinements were performed with use of the Levenberg-Marquardt algorithm with $\chi^{2}$ minimization parameter.
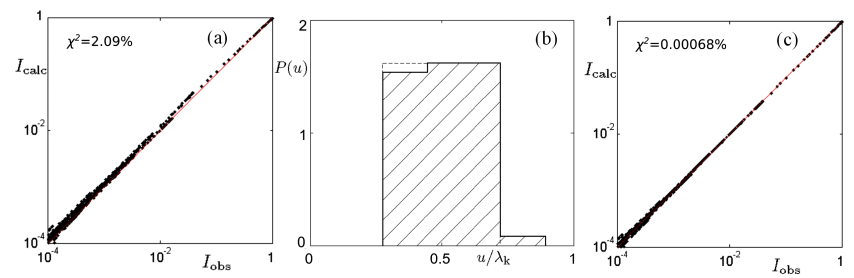

Fig. 5. (a) Log-log plot of calculated vs. observed data for Fibonacci chain with $5 \%$ of phasons in the structure and no correction. (b) The influence of phasons on distribution $P(u)$. If flip mechanism is $L S \rightarrow S L$, some part of an original distribution is missing, whereas new region with the same area appears next to it. Finally, the width and shape of a distribution after phason flips is modified (solid line) in compare to original one without flips (dashed). The flip ratio $\alpha=5 \%, \lambda_{k} \approx 1.38 \AA$. (c) The same plot as in (a) but corrected with cardinal sine functions (4). The agreement factors are given in the plots. If the same structure was refined with standard $\mathrm{D}-\mathrm{W}$ factor, the corresponding value of $\chi^{2}$ was $0.88 \%$. 
The use of cardinal sine functions is straightforwardly justified within the statistical approach. Since we know how phasons perturb the shape of the average unit cell $P(u)$, we can re-modify $P(u)$ in the structure factor formula (3). According to Fig. 5b we consider three regions of the distribution $P(u)$, out of which all are flat. The Fourier transform of a flat distribution is a cardinal sine function, as discussed earlier. Thus, the sum of three functions with different coefficients is needed as presented in formula (4). The correction for phasons can equivalently be made to the distribution $P(u)$ itself, before calculating the diffraction pattern and performing the refinement procedure. We can properly deal with phasonic contribution to diffraction pattern by appropriate modification of the average unit cell. For more realistic cases, when the flipping mechanism as well as the flip ratio are not known, this can be done in different ways: by modelling the distribution with a histogram (choosing small enough bins one can always reproduce the exact shape of the distribution with an expected precision) or by approximating the shape of $P(u)$ with some known function (like Gaussian in classical D-W factor). The first way introduces more parameters than usually. It is however a worth effort because we still waive the multiple fitting procedure. Second way can be successfully applied to 2-dimensional Penrose tiling as model of decagonal quasicrystals [18]. Finally, we can use statistical moments of the distribution as fitting parameters to the diffraction pattern. The latter method was already applied to the Fibonacci chain in our previous paper [21]. It appears that the number of parameters required is not high, several (6-9) parameters are fully enough to restore the shape of the average unit cell satisfactorily well.

\section{Conclusions}

The main purpose of crystallography is to solve and refine crystal structures based on measured diffraction data. Complex crystal structures require big datasets consisting also of weak reflections. By using powerful synchrotron facilities and modern detectors it is possible to collect diffraction patterns with a very high dynamic range. It is, however, a big challenge to properly process the measured data. In our paper we discuss the limitations of the $\mathrm{D}-\mathrm{W}$ factor in terms of structure refinement and propose a way to improve the results of such analysis. We prove that the $\mathrm{D}-\mathrm{W}$ factor substantially limits the range of diffraction data possible to use in a refinement process. It works correctly only for small values of the exponential argument in the above-mentioned formula. For real crystals (including quasicrystals), satisfactorily good results are only obtained for strong reflections with intensities higher than $1 \%$ in relative scale. Peaks with intensities $10^{-4}-10^{-3}$ are refined rather incidentally (see e.g. $[7,8]$ ). This means that including weak reflections in a refinement procedure frequently makes the refinement results worse with lower agreement factor. We mean here that modern refinements with big range of intensities included in the data suffer from systematic deviation observed in small peaks regime, which was also confirmed by our calculations. This phenomenon can be justified by analyzing the influence of weak reflections on the refinement. Weak reflections occur for high values of the $\boldsymbol{k}^{\perp}$, whereas strong ones have small $\boldsymbol{k}^{\perp}$. According to formula (2) proper correction of the strong reflections will result with significant decrease of the calculated weak reflections intensities. Although strong reflections are well corrected the convergence agreement is worse due to large number of weak reflections corrected improperly. The correction in the form of $\mathrm{D}-\mathrm{W}$ factor will succeed only if weak reflections are limited to small value of $\boldsymbol{k}^{\perp}$. We show how to improve the use of D-W factor. Our method based on the average unit cell concept gives a unique tool for proper dealing with phonons and phasons in a fully mathematically correct way.

For 1D quasicrystal we showed that the classical formula for D-W factor (exponential form) satisfactorily well describes phonons in quasicrystals, but is highly ineffective in terms of phasons. We propose new approach to phasonic correction based on statistical method and modeling of the atomic distribution in physical space. Correction for phasons can be done in two ways - whether by applying a multiplicative factor of cardinal sine functions to the structure factor formula or by reshaping of the atomic distribution (equally, redefinition of the structure factor formula) accordingly to the phasonic distortion. In the latter case the correction is made with no iterative refinements of the multiplicative exponential term. For model 1D quasicrystal the statistical method gives much better results of the refinement and significantly improves the agreement factors.

\section{Acknowledgments}

Authors acknowledge financial support from Polish National Science Center under grants no. DEC2013/11/B/ST3/03787 (all) and 2014/13/N/ST3/03776 (R.S.). J.B. also acknowledges the support from Marian Smoluchowski Krakow Research Consortium "MatterEnergy-Future" under KNOW dotation.

\section{References}

[1] K.N. Trueblood, H.-B. Buergi, H. Burzlaff, J.D. Dunitz, C.M. Gramaccioli, H.H. Schulz, U. Shmueli, S.C. Abrahams, Acta Crystallogr. A 52, 770 (1996).

[2] M. de Boissieu, Philos. Mag. 88, 2295 (2008).

[3] M. de Boissieu, Chem. Soc. Rev. 41, 6778 (2012).

[4] M. de Boissieu, Philos. Mag. 86, 1115 (2006).

[5] T.C. Lubensky, Aperiodicity and Order, Ed. M.V. Jaric, Vol. 1, Academic Press, London 1988.

[6] T. Janssen, A. Janner, Acta Crystallogr. B 70, 617 (2014).

[7] P. Kuczera, B. Kozakowski, J. Wolny, W. Steurer, J. Phys. Conf. Ser. 226, 01200 (2010).

[8] P. Kuczera, J. Wolny, F. Fleischer, W. Steurer, Philos. Mag. 91, 2500 (2011).

[9] P. Kuczera, J. Wolny, W. Steurer, Acta Crystallogr. B 68, 578 (2012). 
[10] J. Wolny, B. Kozakowski, P. Kuczera, L. Pytlik, R. Strzałka, A. Wnek, Acta Phys. Pol. A 126, 625 (2014).

[11] J. Wolny, Philos. Mag. A 77, 395 (1998).

[12] P. Buczek, L. Sadun, J. Wolny, Acta Phys. Pol. B 36, 919 (2005).

[13] J. Wolny, B. Kozakowski, P. Kuczera, L. Pytlik, R. Strzalka, Aperiodic Crystals, Eds. S. Schmidt, R. Withers, R. Lifshitz, Springer, Dordrecht 2013, p. 125.

[14] J. Wolny, P. Kuczera, R. Strzalka, Appl. Phys. Lett. 106, 131905 (2015).

[15] B. Kozakowski, J. Wolny, Acta Crystallogr. A 66, 489 (2010).
[16] R. Strzalka, I. Buganski, J. Wolny, Acta Crystallogr. A 71, 279 (2015).

[17] J. Wolny, A. Wnek, J.L. Verger-Gaugry, J. Comput. Phys. 163, 313 (2000).

[18] J. Wolny, B. Kozakowski, P. Kuczera, R. Strzalka, A. Wnek, Isr. J. Chem. 51, 1275 (2011).

[19] J. Wolny, I. Buganski, R. Strzalka, J. Appl. Cryst., submitted for publication.

[20] I. Buganski, R. Strzalka, J. Wolny, Acta Phys. Pol. A 130, 833 (2016).

[21] I. Buganski, R. Strzalka, J. Wolny, Phys. Status Solidi B 253, 450 (2015). 